Edición Extraordinaria. p.p. $157-164$

Memorias del VIII Encuentro Nacional de Experiencias en Enseñanza de la Biología y la Educación Ambiental. III Congreso Nacional de Investigación en Enseñanza de la Biología.

\title{
CONJUNTO DE SIGNIFICADOS DEL CUESTIONARIO DE OPINIONES SOBRE CIENCIA, TECNOLOGIA Y SOCIEDAD (COCTS) EN LA FORMACIÓN CONTINUA DE DOCENTES DE EDUCACIÓN INICIAL Y EDUCACIÓN BÁSICA PRIMARIA MEDIANTE LA CLASIFICACIÓN MÚLTIPLE DE ÍTEMS ${ }^{1}$
}

\section{Resumen}

A continuación se presentan los resultados parciales del Cuestionario Múltiple de Items (CMI), primer instrumento aplicado a un grupo de cuatro profesoras de Educación Inicial y Básica Primaria, como parte del proyecto de tesis doctoral. El instrumento se centró como medio para hacer comparaciones de las concepciones sobre cienciatecnología-sociedad-ambiente en el grupo de las profesoras participantes de una convocatoria de cualificación y formación continua en el marco interinstitucional de cooperación 129 de 2014 entre el Instituto para la Investigación Educativa y el Desarrollo Pedagógico (IDEP) y el grupo de investigación: Construcción de Conocimiento Científico desde la Perspectiva de los Enfoques Didácticos, perteneciente al Doctorado Interinstitucional de la Universidad Pedagógica Nacional (UPN). El análisis de la información se realizó a través del software HUDAP8, cuyos resultados evidencias las tendencias de las profesoras hacia cinco regiones que se identifican con alguna(s) de las cuatro categorías taxonómicas: Definiciones de ciencia y tecnología; sociología externa de la ciencia; sociología interna de la ciencia; y epistemología (Vásquez, 2014).

B

I

0

$\mathbf{P}$

0

$N$

E

N

C Valencia Valbuena Fredy

Estudiante Doctorado Interinstitucional en Educación- Universidad Pedagógica Nacional

Grupo de investigación: Construcción de Conocimiento Científico desde la perspectiva de los enfoques Didácticos.

Docente Secretaria de Educación de Bogotá correo: frevalva@yahoo.com

Then the partial results of Multiple Items Questionnaire (CMI), first instrument applied to a group of four teachers of Early Education and Basic Primary as part of the doctoral thesis are presented. The instrument focused as a means for comparison of the conceptions of science-technology-society-environment group of teachers participating in a call for qualification and continuous training in the interagency cooperation framework 129 of 2014 Research Institute Educational and Pedagogical Development (IDEP) and the research group: Construction of Scientific Knowledge from the Perspective of Educational Approaches belonging to the Inter Doctorate of the National Pedagogical University (UPN). The data analysis was performed using software HUDAP8, the results evidence the trends of the teachers to five regions identified with a $(s)$ of the four taxonomic categories: definitions of science and 
Memorias del VIII Encuentro Nacional de Experiencias en Enseñanza de la Biología y la

Educación Ambiental. III Congreso Nacional de Investigación en Enseñanza de la Biología.

technology; external sociology of science; internal sociology of science; and epistemology (Vasquez, 2014).

Palabras clave: Cuestionario múltiple de ítems (CMI), Cuestionario de opiniones sobre ciencia, tecnología y sociedad (COCTS), y taxonomía

Keywords: Multiple questionnaire items (CMI), Questionnaire views on science, technology and society (COCTS), and taxonomy.

\section{Introducción}

En los últimos años se han origina debates en torno al conocimiento de los profesores, en particular el conocimiento escolar en las clases de ciencias en Básica Primaria (Martínez y Molina, 2009). En este sentido, es necesario realizar investigaciones que contribuyan en indagar el conocimiento profesional de los profesores, particularmente en profesores de la Educación Inicial y Básica Primaria que abordan la enseñanza de las ciencias.

A continuación se presentan los resultados del Cuestionario Múltiple de Items (CMI), primer instrumento aplicado a un grupo de cuatro profesoras de Educación Inicial y Básica Primaria, desde un proyecto de tesis doctoral. El instrumento se centró sobre la concepción de las profesoras participantes y su análisis a partir del uso del software HUDAP8, como medio para hacer comparaciones desde el Cuestionario Múltiple de Items (CMI) técnica para recolección de información (Páramo, 2008). Luego se hace una aproximación con referencias teóricas. Las categorías fueron analizadas en relación con la densidad de similitudes que el software arroja. Para hacer la aproximación teórica se relaciona con ocho taxonomías: 1. Ciencia y Tecnología; 2. Influencia de la Sociedad sobre la Ciencia/Tecnología; 3. Influencia de Ciencia/Tecnología sobre la Sociedad; 4. Influencia de la ciencia escolar sobre la Sociedad; 5. Características de los científicos; 6. Construcción social del conocimiento científico; 7. Construcción social de la Tecnología; y 9. Naturaleza del conocimiento científico, propuestas desde los temas ciencia-tecnología-sociedad-ambiente (Vásquez, 2014).

\section{METODOLOGIA}

Se empleó la clasificación Múltiple de Ítems como técnica de tipo cualitativo de recolección de información (Páramo, 2008), siendo un tipo de entrevista semiestructurada de análisis cualitativo. Para llevar a cabo la aplicación de la CMI se explicitó la técnica a las profesoras, quienes clasificaron en tres momentos las 
Memorias del VIII Encuentro Nacional de Experiencias en Enseñanza de la Biología y la Educación Ambiental. III Congreso Nacional de Investigación en Enseñanza de la Biología.

veintiocho tarjetas que contenían conceptos sobre Ciencia-Tecnología-SociedadAmbiente (Vásquez, 2014).

Durante los tres momentos (inicial) para hacer la primera conformación de sub grupos y acercarnos a los puntos de vista acerca de la naturaleza de la ciencia NdCyT; (intermedio) para generar que existen múltiples alternativas de clasificación; y el (final) para provocar reflexiones de los puntos de vista iniciales. El instrumento diseñado corresponde con la técnica de Cuestionario Múltiple de Items (CMI) "es un tipo de entrevista individual que se fundamenta en el surgimiento libre y espontáneo de criterios por parte del entrevistado para realizar clasificaciones cualitativas sobre un tema en particular..." (Paramo, 2008. pp. 161). En cuanto a la metodología para el instrumento fue: el entrevistado conforma con las veintiocho tarjetas los subgrupos que considere, le asigna un nombre a cada subgrupo, establece un criterio en común para todos los subgrupos y finalmente puede hacer observaciones a esta clasificación, nuevamente realiza el ejercicio hasta tener tres clasificaciones en tres momentos. En relación con la técnica de análisis de datos fue: identificación de las categorías significativas, clasificación de las categorías y subcategorías, identificación de los subgrupos; triangulación de los tres momentos. En correspondencia con los pasos para el análisis de datos fueron: introducir los tres momentos del CMI de cada profesora en el software HUDAP8, el cual genera una gráfica de correlación y densidad de las tarjetas, con ello se hace la identificación de las subcategorías más densas para posteriormente acercarnos a la categoría para triangular desde el análisis cualitativo con referencias teóricas próximas propias de la Investigación-acción, en particular con los temas Ciencia-Tecnología-Sociedad-Ambiente (Vásquez, 2014), el conocimiento profesional del profesor y la cualificación permanente desde la construcción del conocimiento.

La muestra de investigación correspondió a cuatro casos $(A, B, C$ y $D)$ con tres profesoras de primera infancia (pre-jardín) y una profesora de quinto de primaria. Las cuatro profesoras pertenecen a dos instituciones educativas públicas de Bogotá y quienes se inscribieron libremente en una convocatoria abierta a profesores de todos los niveles de la Secretaria de Educación de Bogotá y fueron seleccionadas para participar. Así, la participación fue consentida y concertada con cada una de las participantes, mediante la convocatoria "Reflexionemos y Sistematicemos nuestras prácticas. El Profesor de Ciencias como sujeto de conocimiento" como estrategia de acompañamiento y formación a 15 maestros de aula de 12 colegios distritales de Bogotá.

\section{ANÁLISIS DE INFORMACIÓN}

Esta información se analizó mediante un Análisis de Escalogramas Multidimensionales (MSA, por su sigla en inglés), por medio del software HUDAP8, este programa que fue alimentado por una matriz sobre las distintas clasificaciones que hicieron los profesores, donde la fila principal lleva el código creado (ejemplo, P8C2), la primera 
Edición Extraordinaria. p.p. $157-164$

Memorias del VIII Encuentro Nacional de Experiencias en Enseñanza de la Biología y la Educación Ambiental. III Congreso Nacional de Investigación en Enseñanza de la Biología.

columna el número de la tarjeta, y las demás columnas el número del sub grupo creado por el docente. El programa arrojó como resultado un gráfico que muestra en un espacio bidimensional una serie de puntos que representan los conceptos que fueron objeto de la entrevista por cada uno de los profesores (Ver Gráfico 1).

El diagrama que se presenta en el grafico 1 corresponde a la distribución de las 28 tarjetas propuestas por las 4 profesoras de educación inicial y básica primaria, en un espacio en el que la cercanía entre una y otra tarjeta indica su grado de correlación. Las cercanías espaciales entre una y otra categoría indica su grado de correlación. A partir de la distribución espacial de las categorías se crearon regiones mediante trazos para hacer la interpretación, con la intensión de generar sentido a estas agrupaciones o correlaciones entre categorías. En el diagrama emitido por el software HUDAP8 se pueden identificar cinco regiones los cuales van a ser interpretados con las 8 taxonomías de Vásquez, (2014) (ver tabla 1) con la intensión de dar sentido a estas correlaciones de tarjetas, así en el diagrama emitido del análisis procesado por el software HUDAP8.

Tabla 1. Taxonomías de los temas ciencia-tecnología-sociedad-ambiente

\begin{tabular}{|l|l|}
\hline \multicolumn{1}{|c|}{ TAXONOMIAS } & \multicolumn{1}{c|}{ TARJETAS } \\
\hline 1. Ciencia y tecnología, & 2,3 Y 4 \\
\hline 2. Influencia de la Sociedad sobre la Ciencia/Tecnología & $8,17,19,20$ Y 27 \\
\hline 3. Influencia de la Ciencia/Tecnología sobre la sociedad & $11,16,18,21,28,24$ Y 28 \\
\hline 4. Influencia de la ciencia escolar sobre la sociedad & 13 \\
\hline 5. Características de los científicos & 22 Y 25 \\
\hline 6. Construcción social del conocimiento & 26 \\
\hline 7. Construcción social de la tecnología & 1 \\
\hline 8. Naturaleza del conocimiento científico & $5,6,7,9,10,12,14$, Y 15 \\
\hline
\end{tabular}

\section{RESULTADOS Y DISCUSIÓN}

Se recopilaron todos los criterios utilizados, con libertad y espontaneidad, por las cuatro profesoras para clasificar las veintiocho tarjetas según su correspondencia y densidad. El (gráfico 1) presenta la distribución de las variables (categorías que emergieron del análisis de contenido), las cuales permiten develar el conocimiento profesional que tienen las profesoras entorno a la naturaleza de las ciencias ( $\mathrm{NdC}$ ) desde la ciencia-tecnología-sociedad-ambiente. 
Edición Extraordinaria. p.p. 157 - 164

Memorias del VIII Encuentro Nacional de Experiencias en Enseñanza de la Biología y la

Educación Ambiental. III Congreso Nacional de Investigación en Enseñanza de la Biología.

Grafico 1. Resultados del software HUDAP8 sobre los 12 criterios de clasificación de las cuatro profesoras de educación Inicial y Básica Primaria

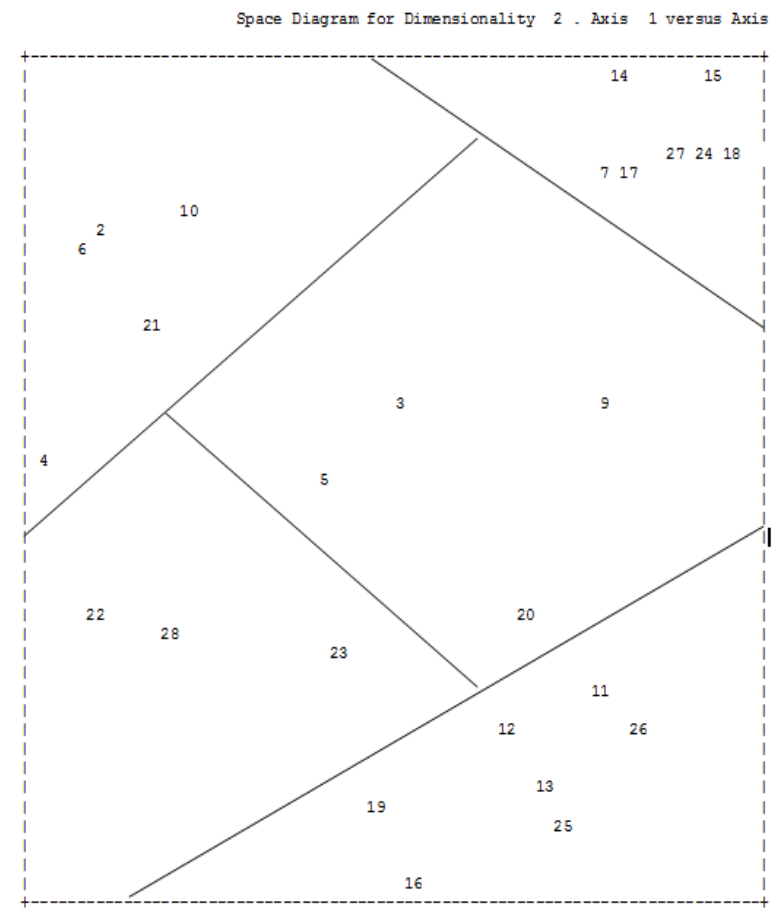

El diagrama que se presenta corresponde a la distribución de las 28 tarjetas en las 12 categorías propuestas por las 4 profesoras, con un criterio y es que el espacio de cercanía entre una y otra tarjeta indica su grado de correlación, Es de aclarar que se está analizando este instrumento y solamente es una aproximación. Pero que de igual manera refleja las tendencias de los doce momentos en los cuales las profesoras realizaron el CMI.

Teniendo en cuenta que el programa asigna un Id a cada tarjeta (ver tabla 2), siendo una idea clave para realizar los análisis correspondientes se debe asimilar estas condiciones como medio para la interpretación de la información.

Tabla 2. Correlación Id con la tarjeta

\begin{tabular}{|l|c|c|}
\hline PALABRA & Número de tarjeta & Id grupo 1 \\
\hline APLICACIÓN CIENTÍFICA & 1 & 15 \\
\hline FISIÓN & 2 & 14 \\
\hline
\end{tabular}


Edición Extraordinaria. p.p. $157-164$

Memorias del VIII Encuentro Nacional de Experiencias en Enseñanza de la Biología y la Educación Ambiental. III Congreso Nacional de Investigación en Enseñanza de la Biología.

\begin{tabular}{|l|c|c|}
\hline PRUEBAS & 3 & 18 \\
\hline FUSIÓN & 4 & 7 \\
\hline TEORİA ATÓMICA & 5 & 27 \\
\hline DISCIPLINAR & 6 & 11 \\
\hline LABORATORIO & 7 & 24 \\
\hline POLÍTICA & 8 & 5 \\
\hline MULTIDISCIPLINAR & 9 & 13 \\
\hline CIENTÍFICOS & 10 & 17 \\
\hline DESARROLLO TECNOLÓGICO & 11 & 3 \\
\hline ÁTOMOS & 12 & 20 \\
\hline ESCUELA & 13 & 12 \\
\hline AVANCE CIENTÍFICO & 14 & 9 \\
\hline RADIACIÓN & 15 & 2 \\
\hline JAPÓN & 16 & 1 \\
\hline ROOSEVELT & 17 & 16 \\
\hline CAUSAS & 18 & 6 \\
\hline EINSTEIN & 19 & 26 \\
\hline HEISENBERG & 20 & 25 \\
\hline SOCIEDAD & 21 & 19 \\
\hline HOMBRES & 22 & 22 \\
\hline VIDA & 23 & 23 \\
\hline MUERTE & 24 & 4 \\
\hline MUJERES & 25 & 28 \\
\hline SEGUNDA GUERRA & 26 & 10 \\
\hline EEUU & 27 & 8 \\
\hline EFECTOS & 28 & \\
\hline
\end{tabular}

En cuanto al gráfico bidimensional que genera el programa, se identificaron 5 regiones (Ver tabla 2). A partir de la distribución espacial de las categorías se establecieron regiones a través de trazos que realizó el investigador, para hacer la interpretación a partir de marcos de referencias, en particular desde Vásquez, (2014) con la intensión de dar sentido a estas correlaciones de tarjetas, así en el diagrama emitido del análisis procesado por el software HUDAP8, se pueden identificar cinco regiones (ver tabla 2).

Tabla 2. Regiones interpretadas por el investigador.

\begin{tabular}{|l|l|l|l|l|}
\hline REGION & \multicolumn{1}{|c|}{ TARJETA } & TEMAS & $\begin{array}{l}\text { MAYOR } \\
\text { TENDENCIA AL } \\
\text { TEMA }\end{array}$ & TAXONOMIA \\
\hline $\mathrm{I}$ & $\begin{array}{l}\text { Política, átomo, } \\
\text { desarrollo } \\
\text { tecnológico, }\end{array}$ & 2,3 Y 8 & $\begin{array}{l}\text { 8. Naturaleza del } \\
\text { conocimiento } \\
\text { científico }\end{array}$ & Epistemología \\
\hline
\end{tabular}


Edición Extraordinaria. p.p. $157-164$

Memorias del VIII Encuentro Nacional de Experiencias en Enseñanza de la Biología y la Educación Ambiental. III Congreso Nacional de Investigación en Enseñanza de la Biología.

\begin{tabular}{|l|l|l|l|l|}
\hline & $\begin{array}{l}\text { avance } \\
\text { científico }\end{array}$ & & \\
\hline II & $\begin{array}{l}\text { Aplicación } \\
\text { Científica, } \\
\text { fisión, pruebas, } \\
\text { teoría atómica, } \\
\text { fusión, } \\
\text { laboratorio, } \\
\text { científico }\end{array}$ & 7,1 Y 8 & $\begin{array}{l}\text { 1. Ciencia y y } \\
\text { Tecnología } \\
\text { Y 8 Naturaleza del } \\
\text { conocimiento } \\
\text { científico }\end{array}$ & $\begin{array}{l}\text { Definiciones de } \\
\text { Ciencia y } \\
\text { tecnología } \\
\text { Epistemología }\end{array}$ \\
\hline III & $\begin{array}{l}\text { Disciplinar, } \\
\text { multidisciplinar, } \\
\text { escuela, } \\
\text { Roosevelt, } \\
\text { Einstein } \\
\text { Heinseberg, } \\
\text { sociedad }\end{array}$ & $3,4,8$ Y 2 & $\begin{array}{l}\text { 2. Influencia de la } \\
\text { sociedad sobre la } \\
\text { Ciencia/tecnología }\end{array}$ & $\begin{array}{l}\text { Sociología de la } \\
\text { externa de } \\
\text { Ciencia }\end{array}$ \\
\hline $\begin{array}{l}\text { Hombres, Vida, } \\
\text { Mujeres }\end{array}$ & 3 Y 5 & $\begin{array}{l}\text { 5. características } \\
\text { de los científicos }\end{array}$ & $\begin{array}{l}\text { Sociología de las } \\
\text { interna de } \\
\text { ciencias }\end{array}$ \\
\hline V & $\begin{array}{l}\text { Radiación, } \\
\text { Causas, } \\
\text { Muerte, } \\
\text { Segunda } \\
\text { guerra, efectos }\end{array}$ & 6,8 Y 3 & $\begin{array}{l}\text { 3 Influencia de } \\
\text { Ciencia/Tecnología } \\
\text { sobre la sociedad }\end{array}$ & $\begin{array}{l}\text { Sociología } \\
\text { externa de la } \\
\text { ciencia }\end{array}$ \\
\hline
\end{tabular}

\section{CONCLUSIONES}

A través del COCTS se posibilita una amplia posibilidades de ítems, que permite ser seleccionarlos en conjunto a medida de las necesidades de evaluación, siendo flexible y de uso personalizado por los profesores de aula, permitiendo un mapeo completo del campo, en este sentido, el COCTS tiene 8 temas, y 4 subtemas: Definiciones de ciencia y tecnología; Sociología externa de la ciencia; Sociología interna de la ciencia; y epistemología, que son componentes que hacen parte de la naturaleza de la ciencia.

De acuerdo a lo anterior, en la región I se evidencia mayor tendencia hacia la Epistemología.

En la región II se aprecia mayor tendencia hacia las Definiciones de Ciencia y Tecnología, y a la Epistemología.

En la región III la mayor predilección corresponde con la Sociología externa de la Ciencia.

En la región IV predomina mayor tendencia hacia la Sociología interna de las ciencias.

En la región $V$ se encuentra mayor tendencia de la Sociología externa de la ciencia. 
Bio - grafía. Escritos sobre la Biología y su Enseñanza. ISSN 2027-1034

Edición Extraordinaria. p.p. $157-164$

Memorias del VIII Encuentro Nacional de Experiencias en Enseñanza de la Biología y la

Educación Ambiental. III Congreso Nacional de Investigación en Enseñanza de la Biología.

\section{REFERENCIAS BIBLIOGRÁFICAS}

Páramo, P. (2008). La clasificación múltiple de ítems (CMI). En : Páramo, P. (comp). La investigación en las ciencias sociales. Técnicas de recolección de información. (pp. 161-170). Bogotá, D.C.; Universidad Piloto de Colombia: Net Educativa

Vásquez, A. (2014). Enseñanza, Aprendizaje y Evaluación en la Formación de Docentes en Educación CTS en el contexto del siglo XXI. Uni-pluri/versidad. 14(2), pp. 3749.

Martínez, C y Molina, A. (2009). El conocimiento profesional de los profesores de ciencias de primaria sobre el conocimiento escolar en el Distrito Capital: Un problema de investigación. Asociación Colombiana para la investigación en Ciencia y Tecnología EDUCYT, memorias, I congreso nacional de Investigación en educación en ciencias y tecnología. 